\title{
Quand le rite devient technique
}

Sacrifice et abattage rituel dans le monde musulman

When ritual becomes tecnology. Sacrifice and rital slaughter in the Muslim world

\section{Pierre Bonte}

\section{(2) OpenEdition Journals}

Édition électronique

URL : https://journals.openedition.org/tc/5020

DOI : $10.4000 /$ tc. 5020

ISSN : 1952-420X

Éditeur

Éditions de l'EHESS

\section{Édition imprimée}

Date de publication : 30 juin 2010

Pagination : 547-561

ISSN : 0248-6016

Référence électronique

Pierre Bonte, "Quand le rite devient technique », Techniques \& Culture [En ligne], 54-55 | 2010, mis en ligne le 30 juin 2013, consulté le 29 septembre 2022. URL : http://journals.openedition.org/tc/5020 ; DOI : https://doi.org/10.4000/tc.5020 


\section{QUAND LE RITE DEVIENT TECHNIQUE}

\section{Sacrifice et abattage rituel dans le monde musulman}

in Techniques et culture 21, 1993: 79-96

Les médiations techniques entre l'homme et l'animal occupent une place un peu particulière dans l'analyse ethnologique des techniques. Leur étude s'inscrit dans un écart maximal par rapport aux méthodes d'analyse introduites par André Leroi-Gourhan qui, ainsi que le remarquait déjà J.-P. Digard ${ }^{1}$, avait essentiellement conjugué ses efforts sur les techniques impliquant la matière dans son ordre physique ou chimique, à l'exclusion ${ }^{2}$ de la matière vivante. Il faut ajouter, ce qui est une autre manière de dire la même chose, que cette relation entre l'homme et l'animal domestique est pauvre en outils spécialisés et beaucoup plus construite en fonction de connaissances et de savoirs, sinon d'affects et de passions.

Cet écart maximal par rapport aux méthodes d'analyse initiées par Leroi-Gourhan me semble paroxystique quand il s'agit de s'interroger sur la notion de tendance telle qu'il la définit. Pour une part essentielle à mes yeux, les effets de convergence et de détermination dont relève la notion de tendance sont liés aux lois physiques et chimiques qui organisent cette matière « inerte » inspirant prioritairement à Leroi-Gourhan ses analyses technologiques. Le traitement de la matière vivante est d'une autre nature, ne serait-ce que parce que l'homme lui-même appartient à son règne. Certes, ce que l'on pourrait appeler par analogie les « lois biologiques » assurent les mêmes effets de convergence et de détermination. C'est en fonction des notions de sexe, d'âge et de filiation que s'organise par exemple universellement la parenté, pour autant, ce qui n'est pas tout à fait faux, que celle-ci soit un système technique de reproduction de la vie humaine. Mais la particularité d'un tel « système », il n'est guère, je pense, nécessaire d'insister là-dessus, c'est que dès le départ activité « technique » et activité symbolique y 
sont intimement et indissociablement liées parce qu'interférent de multiples médiations de pouvoir, de domination, d'appropriation, d'identification, et les représentations qu'elles impliquent, au point que l'aspect « technique ", à juste titre, s'estompe et disparaît, ne ressurgissant sur le plan théorique qu'à travers les multiples avatars des fonctionnalismes.

C'est dans ce contexte, longuement évoqué par Digard dans son ouvrage consacré à la domestication animale (1990), que se situe la médiation technique entre l'homme et l'animal. L'appartenance de l'homme au règne biologique et la place de l'animal dans la chaîne alimentaire se traduisent en outre par de constants transferts des représentations de l'homme à l'animal, et réciproquement. Est-ce à dire que la notion de tendance, telle qu'elle a été élaborée par Leroi-Gourhan, doit être abandonnée? Il me semble, au contraire, qu'elle peut garder sa fécondité anthropologique au sens vrai du terme, qui implique la convergence et la comparaison. Pour ce faire, il faut la débarrasser des présupposés positivistes que l'importance première accordée par Leroi-Gourhan aux lois physiques et chimiques contribue parfois à lui imputer, en partie à tort d'ailleurs. Ce débat me semble traverser le champ de l'analyse ethnologique des techniques depuis l'œuvre fondatrice de cet auteur ${ }^{3}$. Lanalyse de certains faits techniques concernant le phénomène de la domestication animale peut concourir, comme le pense aussi Digard, à un déplacement des conditions de cette analyse qui soulignent, dès le niveau de la tendance, le poids des représentations, de l'activité symbolique et de lois qui seraient d'« ordre psychologique ${ }^{4}$. Un tel déplacement aurait-il réellement choqué l'auteur du Geste et la Parole?

Dans ce trop vaste domaine de la domestication animale je focaliserai mon attention sur un thème particulier, la mise à mort de l'animal, moment clé, tant du fait des représentations qui pèsent sur cet acte technique, fortement chargé sur le plan affectif et émotif, que de son insertion dans la chaîne technique entre le temps de l'acquisition et celui de la consommation. Mis à mort, l'animal est transformé en carcasse bonne à manger, à l'exclusion d'autres types d'usages de l'animal vivant ${ }^{5}$, choix économique mais aussi social et culturel qui sépare dans les sociétés pastorales et agro-pastorales l'élevage pour le lait et l'élevage pour la viande. Ce moment a été moins étudié ${ }^{6}$ que d'autres aspects du processus technique de l'abattage, comme le partage des carcasses, la découpe, qu'il précède pourtant et détermine. Il renvoie cependant à un problème classique de l'anthropologie, depuis W. Robertson Smith ou encore Henri Hubert et Marcel Mauss, celui de la place du phénomène sacrificiel, il est vrai bien rarement étudié du point de vue de l'analyse ethnologique des techniques, malgré mes modestes efforts en ce sens (Bonte 1984 et 1985) après ceux d'autres auteurs plus compétents.

Dans de très nombreuses sociétés, en effet, des auteurs, dont l'intérêt portait d'abord sur la dimension rituelle du sacrifice, ont remarqué que la mise à mort de l'animal ne pouvait avoir lieu que dans un contexte rituel et n'obéissait pas - du moins les masquait-elles avec beaucoup de force - à des motivations d'ordre alimentaire.

E.E. Evans-Pritchard, par exemple, a souligné dans des pages remarquables consacrées au sacrifice chez les Nuer (1956) que la finalité de l'élevage des bovins dans cette société, outre de disposer des biens cérémoniels utilisés à l'occasion du mariage et d'autres rites sociaux, était d'avoir des bêtes à sacrifier dans les multiples occasions rituelles prévues dans leur vie sociale et culturelle. Tuer une bête aux seules fins de s'en nourrir est impensable pour les Nuer et les mythes expliquent comment et pourquoi le bétail a été donné aux hommes, et non aux femmes par exemple, à cette fin. Dans la Grèce ancienne, comme dans l'ensemble du monde indo-européen, le sacrifice est aussi la 
seule occasion de tuer un animal et la «cuisine du sacrifice » fournit en viande la communauté (Détienne et Vernant 1979), si bien que le vocabulaire ne distingue pas entre le tueur-boucher et le sacrificateur (mageiros). Ces textes, et ces faits, sont bien connus, et je n'y insisterai pas. Ils posent un problème général, sinon universel - est-il de mise à mort de l'animal domestique autre que rituelle et masquant les finalités alimentaires? - parfois posé de manière plus générale encore : la motivation première de la domestication animale, dans les sociétés concernées, n'est-elle pas finalement et originellement d'ordre religieux? Il n'est pas de notre propos de reprendre ces problèmes, du moins pouvons-nous remarquer que nous sommes là dans quelque chose qui est de l'ordre de la tendance. Mais celle-ci n'est plus alors d'essence positive, elle relève exclusivement des représentations que se font les sociétés de la place de l'animal et plus généralement de l'ordre du monde ${ }^{7}$.

Précisant encore mes investigations je procéderai à un nouvel effet de focalisation pour me concentrer désormais sur le seul sacrifice musulman. Ce choix n'est pas seulement conditionné par le déroulement de mes propres recherches ${ }^{8}$, il répond aussi aux caractères spécifiques de l'abattage comme fait technico-rituel dans les sociétés musulmanes. Par certains côtés, le sacrifice musulman entre dans cette catégorie générale, précédemment introduite, des sociétés où tout abattage d'un animal est rituel, cependant la finalité proprement sacrificielle de l'abattage n'est évoquée qu'en certaines circonstances, et encore de manière assez ambiguë - il n'existe pas en fait de terme recouvrant

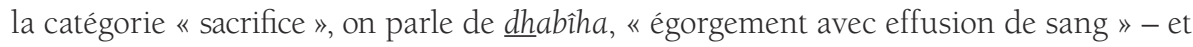
le rituel d'abattage à des fins exclusivement alimentaires est souvent réduit à une simple codification de l'acte d'égorgement sans impliquer les temps, les espaces, ni les individus spécialisés qui composent habituellement la trame de l'acte sacrificiel. L'abattage rituel produit une viande consommable car licite (halâl) et l'intention sacrificielle est souvent absente. Cet abattage est sans aucune difficulté susceptible de s'inscrire, comme l'abattage casher chez les Juifs, dans une société moderne et technicisée qui produit de la viande halâl, en vente dans les supermarchés, simple marqueur d'identité d'un groupe, Musulmans ou Juifs, qui exprime son particularisme à travers ce type de pratiques.

\section{Du beau sacrifice à l'abattage halâl}

La simple lecture du Coran et des Hadîth prouve amplement que le sacrifice n'occupe pas une place centrale dans l'islam dont il n'est d'ailleurs pas l'un des « piliers »9. Quand il est évoqué dans les textes sacrés, il relève de la «tradition », qu'assume le Prophète en définissant les règles du sacrifice durant le Pèlerinage, ou en évoquant le sacrifice qui accompagne les rituels du cycle de vie (naissance, imposition du nom, rasage des cheveux, autrefois peut-être circoncision, rassemblés dans le sacrifice 'aqîqa). Par contre, la codification rituelle de l'abattage des animaux est d'inspiration divine, révélée par le livre de l'appel ${ }^{10}$. Il existe pourtant un modèle sacrificiel, sans que l'on puisse établir de différences significatives, dans son aspect performatif, par rapport aux règles relativement simples de l'abattage rituel. Les principales différences résident au niveau de l'intentionnalité de l'acte, telles qu'elles se traduisent dans le vocabulaire. 


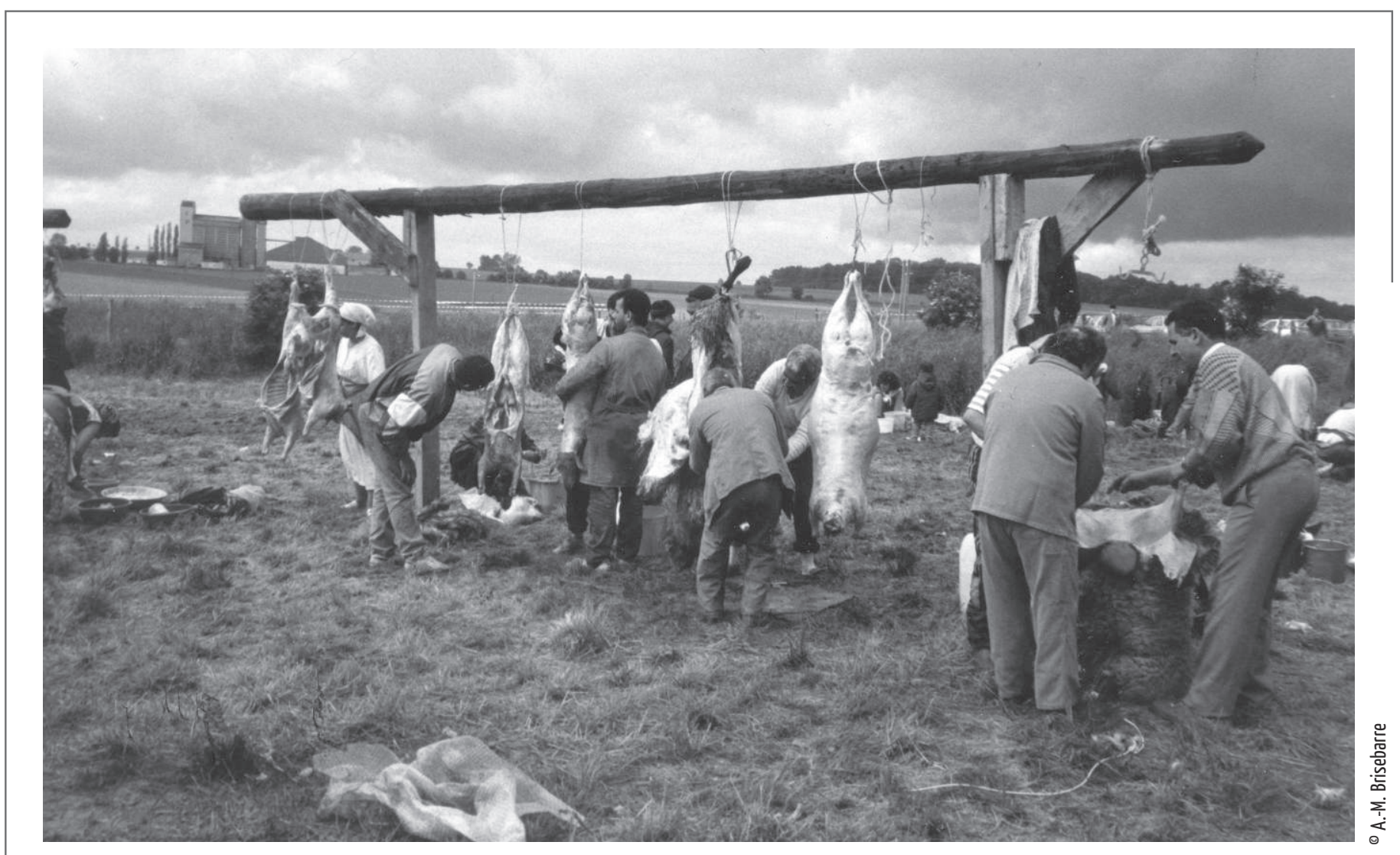

France, Ayd, 1994.

Le sacrifice est effectué sur un site dérogatoire par rapport à la réglementation nationale et européenne, ici une ferme.

\section{Le « beau sacrifice »}

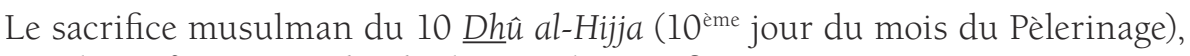
appelé parfois yawm al-nahr, le jour du sacrifice, occupe une position importante dans le dispositif symbolique qui entoure le sacrifice. Outre celui réalisé à cette date par les pèlerins à La Mecque, l'ensemble des Musulmans célèbrent ce jour-là un sacrifice: c'est la fête de l'cAyd al-kabîr, parfois appelé Tabaski dans les pays musulmans d'Afrique noire.

Ce sacrifice, accompli par le Prophète, qui en a produit le modèle musulman, renvoie bien évidemment à des rites pré-islamiques accompagnant le pèlerinage qui avait lieu dans l'espace sacré de la ville sainte. Muhammed, qui l'effectue au nom de la «tradition », et non en fonction des révélations divines qui constituent le Coran, récupère largement un rite ancien tout en opérant des déplacements significatifs.

Le premier de ces déplacements concerne le temps et l'espace sacrificiel ${ }^{11}$. Le rituel sacrificiel est inscrit dans les règles musulmanes du pèlerinage. Il a lieu en des places sans doute assez semblables à celles où se déroulait le sacrifice avant l'Hégire, dans la ravine de Mina, suivant la station qu'effectuent les pèlerins au Mont Arafat, avant la 
lapidation symbolique des démons qui semble, elle aussi, reprise du rite pré-islamique ${ }^{12}$. Les modifications introduites par l'islam sont ailleurs; elles soulignent la subsomption du rituel aux principes de la révélation.

Les textes sacrés indiquent que le sacrifice de ce jour du pèlerinage doit être effectué après la prière du matin; sinon, il est sans valeur. Le changement terminologique enregistre cette évolution: alors que le terme employé avant l'Hégire était hadî, terme qui peut être encore utilisé de nos jours, il sera plus généralement appelé ensuite dahiya, de la racine arabe DHW qui veut dire « entrer dans le jour » pour signifier que le rituel s'inscrit désormais dans le temps de l'islam, rythmé quotidiennement par les prières.

Le rituel sacrificiel est par ailleurs replacé dans le contexte biblique auquel se réfère l'islam, dernière des religions révélées du Livre. Il est conçu comme la répétition du geste d'Abraham (Ibrahim), qui aurait, en ces mêmes lieux, ainsi chargés d'une nouvelle sacralité, organisé le sacrifice de son fils Ismâcîl au Dieu, celui-ci remplaçant, à l'ultime moment, ce sacrifice humain par celui d'un mouton. Dans l'ensemble du monde musulman, de la 'umma, depuis lors, en ce jour de $l^{x}$ Ayd al-Kabîr, des centaines de milliers de moutons, ou d'autres animaux, sont simultanément sacrifiés pour commémorer l'offrande faite par Abraham de son fils à Dieu, fête familiale, à forte fonction identitaire, qui représente un moment fort du culte.

Il existe donc bien un modèle sacrificiel dans l'islam, mais curieusement rapporté, laissant en suspens l'idée de sacrifice, dont s'empareront tous les syncrétismes sans lui accorder une place centrale dans le modèle théologique que produit le Coran avec lequel, nous y reviendrons, elle n'est pas sans entretenir certaines contradictions. Ce modèle, cependant, ne présente aucune caractéristique autre que celles prescrites pour l'abattage rituel, en dehors du fait d'être inscrit dans le temps et l'espace particulier du Pèlerinage. Pratiquement muets sur le sacrifice, les textes sacrés sont en effet prolixes s'agissant de l'abattage rituel, évoqué en particulier dans plusieurs importantes sourates ${ }^{13}$. Voyons maintenant le contenu de ces prescriptions que l'on peut regrouper en trois chapitres.

Les prescriptions portent d'abord sur la nature des animaux abattus. Certains sont strictement interdits, le porc essentiellement, en l'occurrence, mais les Hadith ont progressivement étendu ce champ à l'âne domestique par exemple, ou aux carnassiers, alors que d'autres animaux sont simplement tolérés: le cheval, le lézard, etc. L'exégèse des textes a aussi permis de préciser le mode de catégorisation des animaux non seulement acceptables mais encore conseillés pour le sacrifice et l'abattage rituel. En sont exclus d'abord les animaux borgnes, boiteux, malades ou mutilés. L'âge et le sexe des victimes jouent aussi un rôle important sans pour autant que s'entendent les experts dans les différentes parties de la 'umma. C'est généralement un animal jeune mais parvenu à l'âge adulte qui est considéré comme la victime de choix ${ }^{14}$; les considérations de sexe et celles qui concernent le type d'espèce le plus valorisé sont elles aussi variables dans les limites prescriptives fixées par la parole divine.

Cette dernière accorde une importance toute particulière à l'égorgement et à l'effusion du sang. Non seulement celui-ci ne peut être consommé mais la consommation d'une bête dont le sang n'est pas répandu est interdite; elle est alors assimilée à une charogne, à un cadavre (mayla) immangeable. Les conditions sous lesquelles doit être pratiqué l'égorgement, celles du moment où s'écoule le sang et où meurt l'animal sont aussi prévues avec soin.

La troisième prescription divine et légale concerne la consécration de l'animal à Dieu. Elle est tout aussi importante que les deux premières et mérite tout autant de considérations et de soins. Les modalités anciennes de consécration de la victime peuvent subsister en 
certains cas; s'en rajoutent d'autres dans les formes sacrificielles syncrétiques. Mais la modalité principale de la consécration consiste à invoquer le nom de Dieu (tasmiyyah) ou tout autre formule plus complète en fonction des intentionnalités de l'abattage.

Ces prescriptions, qui définissent le cadre de l'abattage rituel et du sacrifice, obéissent à une logique simple qui vise à définir en termes de licite (halâl) et illicite (harâm) la viande consommée par les Musulmans. Logique binaire qui se subdivise elle-même:

— animaux licites/animaux illicites

— animaux licites consommables/animaux licites non consommables.

Pour comprendre cette logique et les catégories cognitives qui la commandent, il est indispensable d'étudier plus avant les conditions de passage d'une catégorie à l'autre. Elles sont loin de se réduire à une opposition entre le pur et l'impur. Elles débordent en outre le domaine de l'abattage des animaux domestiques pour s'étendre à d'autres domaines faisant intervenir la vie animale, la chasse et la pêche en particulier: les considérations en ces deux domaines éclaireront parfois de manière nouvelle les faits précédemment exposés.

Remarquons d'abord que la définition du licite et de l'illicite est un mode de catégorisation qui déborde largement, dans l'islam, le domaine qui nous concerne, celui de l'abattage rituel, pour s'étendre à des aspects variés de la vie collective et individuelle. Revenons maintenant à la question de l'abattage rituel pour essayer de mettre en évidence les catégories cognitives qui lui sont sous-jacentes.

De ce point de vue, c'est l'attention portée aux modalités de mise à mort de l'animal qui est la plus significative. Le point essentiel est que le sang doit s'écouler de l'animal vivant et être la cause de la mort, quelles que soient les rationalisations qu'ont pu récemment être amenés à mettre en avant les théologiens musulmans sur les avantages techniques de l'égorgement rituel musulman, par rapport à d'autres techniques occidentales plus modernes ${ }^{15}$. Le couteau doit être aiguisé avec soin. La technique d'égorgement est prévue dans le détail: il s'agit de couper simultanément les carotides et la trachée artère sans atteindre la moelle épinière et sans trancher complètement la tête. La position de l'animal est prévue, à cet effet, sur le côté ${ }^{16}$. Il doit en outre être orienté en direction de La Mecque.

La séparation du corps vivant et du sang est le moment clé de l'abattage et nous reviendrons ultérieurement sur la symbolique qu'elle implique. La casuistique musulmane a cependant prévu, souvent dans le détail, les cas où l'acte technique ne peut être effectué dans les règles, en particulier à l'occasion de la chasse où le gibier ne peut être atteint facilement ${ }^{17}$. L'égorgement, même après blessure préalable, reste la méthode préconisée. Il suffit cependant que la blessure reçue et ayant entraîné la mort lors de la fuite de l'animal ait fait couler le sang. L'abattage avec un épieu, une flèche, une arme à feu est ainsi possible, dès lors que le sang coule; par contre, il est interdit de tuer avec des pierres ou d'autres instruments du même type qui assomment, ou encore de consommer un animal étouffé par noyade. Le fait que n'ait pas coulé, même symboliquement, le sang rend la carcasse « illicite » et assimilable à une charogne, à un cadavre impur. La seule mort licite est celle qui sépare le sang du corps. Encore faut-il que celui-ci reste intact. S'il est toléré que la tête soit séparée du corps, sans pour autant que cette technique soit conseillée au moment de la mise à mort, les parties d'un corps séparées de celui-ci, du fait des blessures ou d'attaques de bêtes sauvages par exemple, ne sont pas licites, même si le reste du corps, dès lors que les prescriptions ont été respectées, est lui-même consommable ${ }^{18}$. De même toute mutilation apportée au corps de l'animal vivant est interdite, comme en particulier les sacrifices traditionnels effectués en coupant les jarrets de l'animal avant son égorgement, qui sont pourtant encore pratiqués dans l'aire maghrébine et saharienne. 
L'intentionnalité (niyya) de l'acte technico-rituel de l'abattage joue aussi un rôle majeur. Elle se manifeste d'abord dans la consécration de la victime à la divinité, parfois acquise, comme dans le contexte pré-islamique, par des signes qui marquent sa destination (taqlîd), ou encore par une marque (iscâr) apposée sur l'animal, et qui provoque une blessure et un saignement légers; l'animal ainsi désigné ne peut plus faire l'objet alors d'aucune transaction ni avoir d'autres usages. Avec les mêmes effets, l'islam a remplacé cette consécration par la simple invocation, selon des formules variables, du nom de Dieu. L'intentionnalité de la consécration apparaît plus particulièrement dans les accommodements trouvés en ce qui concerne la chasse et la transformation des animaux sauvages, du gibier, en carcasse. En cas de chasse avec des chiens, la viande rapportée - n’aurait-elle pas été égorgée selon les règles précédemment définies - est consommable si le chien a été intentionnellement dressé à la chasse et si le nom de Dieu a été récité préalablement sur lui. Même chose en ce qui concerne la flèche ou l'épieu, ou encore la balle, qui apportent la mort: le bismillah prononcé au moment de l'utilisation de l'arme rend la viande licite.

Les notions de licite (halâl) et d'illicite (harâm) répondent en définitive, dans leurs grandes lignes, à une catégorisation en termes de pur et d'impur, sans pour autant s'y réduire car si l'impur est dans tous les cas dangereux pour l'homme donc illicite, le pur peut l'être tout autant: la notion de harâm renferme en fait à la fois les notions de pur et d'impur. Les représentations du sang, sur lesquelles je reviendrai, contiennent une telle ambiguité. Aussi est-ce tout autant sur les pratiques elles-mêmes que sur les objets ou les produits que porte ce travail de catégorisation qui aboutit à la définition du licite et de l'illicite.

C'est en ce sens que l'abattage des animaux peut être conçu comme une pratique technico-rituelle - les deux termes étant entendus ici indissolublement liés. Les modalités de l'égorgement, abstraction faite d'ailleurs de l'objet utilisé à cette fin ${ }^{19}$, et la stricte séparation du sang et du corps définissent en effet un processus technique, organisé certes par des représentations que je vais introduire au paragraphe suivant, mais qui, étant abstrait des temps, espaces et circonstances de l'acte rituel, ainsi que de tout personnel rituel spécialisé, peut être reproduit dans les contextes les plus divers comme nous allons le voir maintenant avec l'abattage moderne halâl.

L'intérêt de cette étude de l'abattage rituel halâl ${ }^{20}$ est de montrer l'inscription de la dimension rituelle dans le processus technique décomposé pour répondre aux impératifs d'une logique de type industriel, mais aussi d'introduire des éléments de comparaison avec le processus technique de l'abattage, disons, « occidental » pour simplifier. La comparaison peut s'effectuer presque terme à terme, dans la mesure où les deux systèmes sont soumis aux impératifs de la réglementation européenne en matière d'abattage, modifiée pour tenir compte des spécificités de l'abattage rituel ${ }^{21}$. La distinction entre le licite et l'illicite est soumise dans le cas de l'abattage occidental à une autre distinction, celle du légal et de l'illégal dont nous allons voir, en fait, qu'elle n'est pas foncièrement différente même si ses références sont autres.

L'abattoir industriel organise la chaîne opératoire de l'abattage comme une chaîne industrielle dont les principaux moments et contraintes sont:

- la stricte séparation du bétail vivant de l'animal que l'on tue et des carcasses, entendue ici pour des raisons d'hygiène mais qui correspond en fait aux conditions nécessaires de l'abattage rituel halâl;

— l'acheminement progressif du bétail vers le lieu d'abattage (tapis roulant);

— l'immobilisation de l'animal précédant la mise à mort réalisée au moyen d'un instrument de contention (piège) qui l'amène dans la position d'abattage; cette mesure, 
étendue à l'abattage rituel par les décrets spécifiques qui le concernent, n’apparaît pas, là encore, contradictoire avec la pratique rituelle;

- par contre, celle-ci est en contradiction avec l'obligation légale de l'insensibilisation préalable à la mise à mort (pratiquée au pistolet ou à l'électricité) et en est dispensée; rappelons que l'animal doit être égorgé « vivant » et que l'assommer ou l'étouffer préalablement rendent sa viande illicite.

La principale différence réside donc dans le moment de la saignée qui suit l'insensibilisation et opère sur un animal au statut intermédiaire, pas encore mort mais déjà non vivant, dans l'abattage occidental, alors qu'elle correspond au moment de la mise à mort licite dans l'abattage halâl. Il en résulte des différences d'organisation de la chaîne technique: dans le cas de l'abattage halâl, l'immobilisation de l'animal orienté vers La Mecque, le place dans la position de l'égorgement qui rendra la viande licite; la saignée, ultime et essentiel préalable à la fabrication de la carcasse, intervient dans l'abattage occidental comme un mode de traitement d'un animal déjà « non vivant » du fait des procédures d'insensibilisation.

Lautre différence essentielle concerne, bien sûr, le personnage du « tueur » qui dans l'abattage rituel doit être religieusement agréé, ce qui n'est pas sans poser problèmes s'agissant de la communauté musulmane qui ne possède pas de « clergé » professionnel: tout homme adulte et sain d'esprit, bon musulman de surcroît, y est un sacrificateur en puissance.

Les différences apparaissent en définitive relativement minimes entre ces deux processus techniques et, sous réserve de quelques aménagements, les abattoirs pratiquent souvent les deux modalités d'abattage, en leur réservant respectivement certains jours de la semaine.

\section{Interprétation et conclusion}

Avec les abattoirs rituels halâl nous assistons en quelque sorte à l'achèvement d'un processus de technicisation d'un acte rituel qui a toujours été aussi un acte technique. Situation privilégiée pour examiner plus au fond l'articulation du technique et du symbolique, masquée dans la lourde armature symbolique du sacrifice, tel que l'étudient habituellement les anthropologues, tout autant que dans l'apparente technicité de l'abattage moderne qui réinterprète la dimension rituelle au nom de considérations sanitaires et éthiques ${ }^{22}$.

Dans le monde musulman moderne, le sacrifice lui-même, le beau modèle ibrahimique,

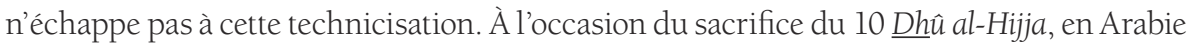
saoudite wahhabite, une banque, la Banque Islamique de Développement (BID), organise les réseaux financiers pour rassembler les contributions qui permettront ce jour-là le sacrifice « par procuration » et la distribution des viandes de près de 500000 ovins ${ }^{23}$. À une autre échelle, dans la Turquie laïcisée moderne, de véritables « maisons du sacrifice » gèrent l'intentionnalité rituelle des actes et la distribution des viandes sacrificielles ${ }^{24}$. Nulle part la mort de l'animal à des fins alimentaires n'est un fait technique aisément gérable. Quel type de gestion en réalise finalement l'islam?

Nous ne pouvons, dans le cadre de cet exposé, reprendre l'ensemble des questions soulevées par la pratique musulmane de cette mort de l'animal telle que nous l'avons jusqu'à présent considérée à travers la catégorisation en termes de licite et d'illicite. Nous nous arrêterons exclusivement sur un point important, que nous retrouvons sous d'autres 


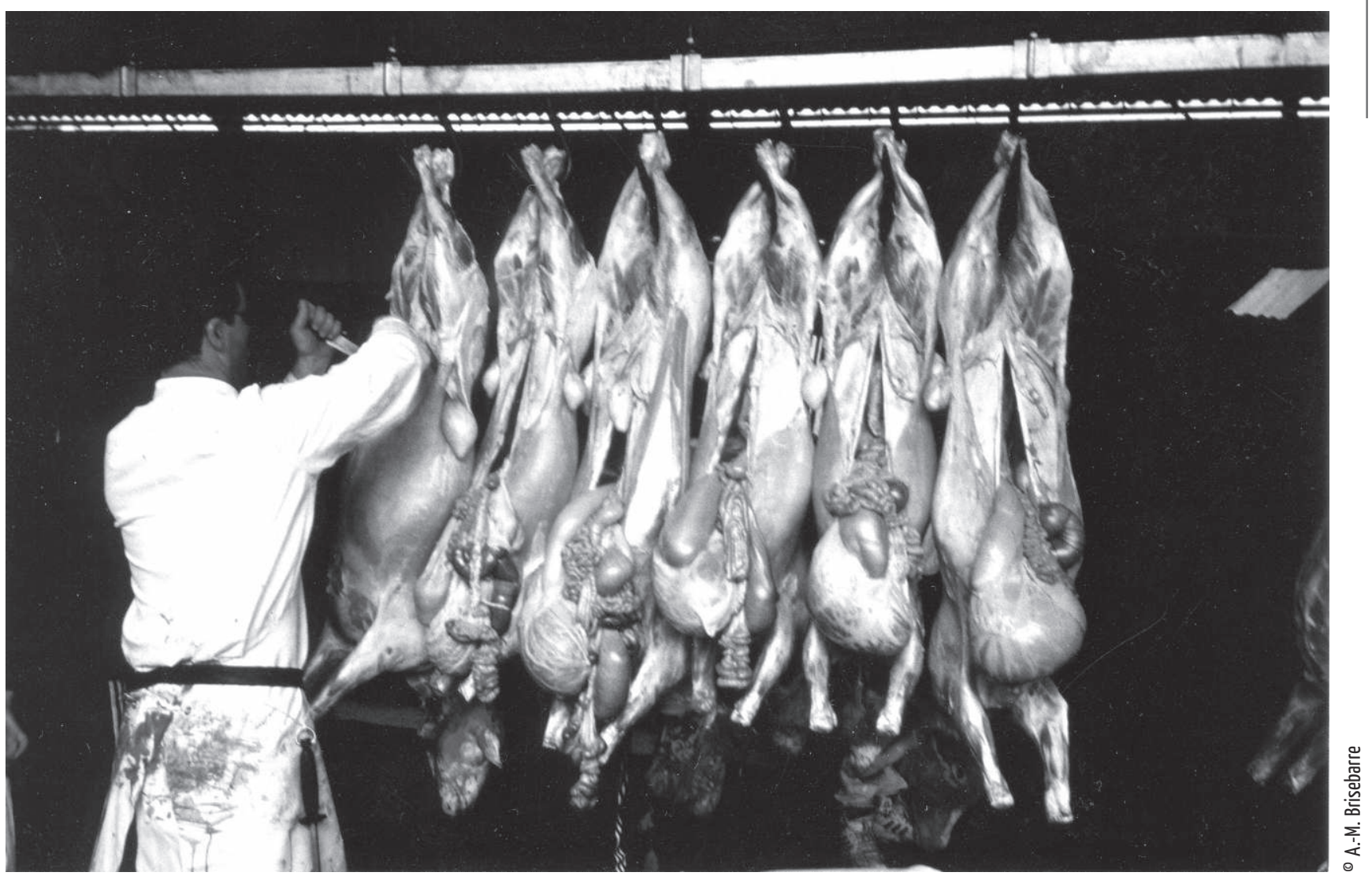

France, Ayd, 1993

Le site dérogatoire est ici un abattoir privé moderne.

codes dans l'abattage « occidental » : la stricte séparation du sang et du corps dans la fabrication de la carcasse, dans la définition du « bon à manger ».

Le terme le plus couramment utilisé pour désigner le sang est en arabe damm, mais on emploie aussi souvent le terme nafs qui désigne aussi l'âme. Dans les conceptions arabes de l'esprit vital, on peut dire en simplifiant quelque peu, que l'âme s'exprime à deux niveaux: un niveau spirituel, parfois assimilé au souffle, que l'on laisse s'exhaler au moment du sacrifice, et un niveau plus végétatif, qui est localisé dans le sang et qui s'exprime avec l'effusion de sang accompagnant et provoquant la mise à mort. Les représentations de l'âme animale ne diffèrent pas de celles de l'âme humaine, l'islam attachant là une grande importance à la vie animale. De ce fait, l'impureté attachée apparemment au sang est d'une grande ambiguité; elle tient plutôt à son caractère sacré et au danger qu'il présente une fois écoulé. De même que le sang humain qui s'écoule est un danger potentiel et un enjeu majeur des relations entre les individus et les communautés - que l'on considère à ce propos la stricte et contraignante codification de la vengeance et du prix du sang-, 
de même le sang versé par l'animal se transforme en une substance dangereuse pour les humains, donc illicite puisque c'est en fonction de ce caractère, beaucoup plus que des notions de pur et d'impur, que se définissent les catégories de licite et d'illicite. Dans les premiers temps de l'islam, on considérait que le sang versé se transformait en un principe dangereux, appelé hâma ou sadâ, parfois assimilé à une petite chouette vivant dans les cimetières (Chelbod 1955) ${ }^{25}$; de nos jours et dans les sociétés maghrébines et sahariennes musulmanes, le sang versé sur le sol, comme les cendres ou les ordures, est le lieu privilégié d'habitat des jnûn et les humains doivent éviter son contact.

On peut noter immédiatement les différences de signification entre la saignée pratiquée lors de l'abattage rituel musulman et celle qui accompagne l'abattage occidental. Dans ce dernier cas, la séparation du sang et du corps est plutôt destinée à éliminer l'animalité, accompagnant, dans le traitement de la carcasse, d'autres pratiques qui nient cette animalité et entraînent, par exemple, un traitement de type végétal de la viande (Vialles 1990). Alors que la mort de l'animal est mise en scène dans le premier cas, entourée de nombreuses précautions qui tiennent à une représentation presque humaine de l'animal, elle est en quelque sorte niée dans le second, les ressemblances biologiques entre l'homme et l'animal étant gommées au maximum pour produire cet écart où la carcasse devient chose bonne à manger.

Revenons à l'abattage rituel musulman, forme valorisée par l'islam alors que, nous l'avons vu, le sacrifice trouve mal sa place dans l'ensemble des pratiques, même si le Prophète reconnaît qu'il est de « tradition ${ }^{26}$. Il est vraisemblable que le sang versé lors du sacrifice jouait avant l'Hégire un autre rôle dans les pratiques religieuses.

W. Robertson Smith (1989) pensait déjà que ce sang était offert à la divinité car il était considéré comme trop sacré pour être mangé: il y aurait ainsi continuité avec le traitement du sang lors de l'abattage rituel ultérieur. Chelhod, de son côté, rapproche les termes dérivés de la racine SDN qui a donné sâdin, le prêtre, et sadîna, le sang, la graisse, pour avancer des hypothèses comparables. Quoi qu'il en soit nous avons d'autres indices qui nous permettent de comprendre quel type de travail symbolique l'islam naissant, et très vite triomphant, a effectué sur la catégorie de sang. Sans interdire vraiment les pactes de sang, qui créaient avant l'Hégire une véritable parenté élective, doublant, comme la parenté de lait, la consanguinité biologique, le Prophète les élimine du champ de la parenté, leur réservant une simple fonction d'alliance (voir Conte 1994). De même le sang sacré du sacrifice est, avant même que celui-ci n'ait été effectué, tout entier entre les mains de Dieu sans qu'il soit nécessaire de le lui apporter comme offrande: « Le sang trouve place auprès de Dieu avant même qu'il n'ait touché terre » déclare une sourate. Une autre sourate, celle du « Pèlerinage », précise que tous les animaux sont destinés et donnés à Dieu, qui ne réclame rien en échange.

De manière générale, l'islam, pour instaurer la nouvelle et exclusive alliance entre les hommes et Dieu, a opéré un travail de sape des catégories symboliques exclusives à travers lesquelles se constituaient auparavant les relations entre les hommes et celles entre les hommes et le surnaturel. Des catégories comme celles de sang, mais aussi de lait, de sperme, etc., semblent avoir joué alors un rôle majeur. Les relations que l'on peut établir dans les représentations entre le lait et le sperme, celles entre le sang et le lait, qui représentent des principes d'alliance, celles encore entre le sang et le sperme que relève Chelhod à travers l'analyse des dérivés de la racine $\mathrm{MNY}^{27}$, peuvent encore être mises en évidence à travers les données actuelles ou les textes anciens. L'étude de ces représentations et de ces catégories nous entraînerait trop loin, j'en garderai simplement l'idée 
qu'elles formaient vraisemblablement un système à visée cosmique, et que ce système était riche de significations que l'islam s'est attaché à travestir, réinterpréter et utiliser au service d'une nouvelle cosmologie monothéiste et d'une nouvelle conception des relations entre les musulmans, entre ceux qui partagent la même foi. Laccent mis sur l'abattage rituel, que rien ne distingue réellement du schéma sacrificiel, sinon l'intentionnalité de l'acte, et le déplacement du système sacrificiel proprement dit hors du champ central de la pratique religieuse s'inscrivent dans ce contexte.

Il est temps de conclure et je le ferai brièvement. Nous sommes partis des notions de tendances et de faits. Je ne récuserai pas, en ce qui me concerne, l'idée de tendance pour classer une série de faits technico-rituels qui relèvent tous de la même attitude de l'homme vis-à-vis de la mort de l'animal domestique. Les représentations divergentes qui sous-tendent la saignée dans l'abattage occidental et dans l'abattage musulman, avec ce qu'elles impliquent quant à l'organisation de la chaîne technique - insensibilisation préalable à la mise à mort et saignée préparant le traitement « désanimalisé » de la carcasse dans un cas, saignée accompagnant la mise à mort dans l'autre qui n'opère pas une coupure radicale entre l'homme et l'animal - ouvrent un champ d'investigation technologique encore peu exploré: les travaux d'inspiration « technologique » sur le sacrifice ont plus insisté jusqu'à présent sur le partage de la carcasse que sur la mise à mort. L'étude des conditions de celle-ci, beaucoup plus que les interrogations sur l'« origine religieuse » de la domestication, peut se révéler féconde, de même que pour aborder cette étude, est féconde, dans sa rigueur comparatiste, la méthode d’approche définie par Leroi-Gourhan.

Ces données impliquent aussi, cependant, quelques mises au point quant aux présupposés théoriques qui peuvent peser sur cette notion de tendance.

Si l'on peut considérer, par analogie, qu'elle relève en ce cas des lois biologiques de la matière vivante - comme relève la matière « inerte » de lois physiques et chimiques -, ceci n'implique nulle détermination de type naturaliste de la tendance, au sens où nous organisons dans notre propre système de pensée la matière vivante. Les représentations et pratiques qui organisent l'abattage occidental, nos propres définitions du licite et de l'illicite s'agissant de cette matière vivante qu'est l'animal, transformé en aliment, soulignent que nos propres pratiques techniques sont elles-mêmes en décalage par rapport à notre système d'appréhension naturaliste, «scientifié ». Que l'on essaie par exemple de faire manger du cheval à un anglais!

Pour être retenue dans l'analyse technologique et s'y révéler féconde, la notion de tendance doit être expurgée de tout présupposé de type positiviste. Il est vraisemblable que les déterminations de types physique et chimique doivent être traitées de la même manière. Sauf à situer la tendance comme une sorte d'ensemble de contraintes générales qui perdent leur valeur heuristique quand il s'agit d'expliquer les faits, en contradiction avec la visée de l'analyse de Leroi-Gourhan, il faut reconnaître que ces contraintes sont dès le départ inscrites et interprétées à travers des systèmes de pensée, des représentations, des catégories dont le langage nous donne l'expression la plus cohérente. Il n'y a pas de savoir-faire sans savoir-dire. Vingt ans après Milieux et Techniques et L'Homme et la Matière, la publication du Geste et la Parole souligne la conscience aiguë qu'avait Leroi-Gourhan de ce problème ${ }^{28}$.

Indissociablement liés, savoir-dire et savoir-faire, peuvent cependant connaître des évolutions divergentes, voire entrer en contradiction: bien des problèmes de changement dans le champ des techniques se posent en ces termes. En gommant, réinterprétant, soumettant à un nouveau système de pensée l'acte technico-rituel de la mise à mort de 
l'animal, l'islam a entraîné une modification conséquente du schéma sacrificiel. Il a en quelque sorte technicisé l'acte rituel qui n'apparaît plus soumis qu'à un savoir-dire d'ordre métaphysique et à des considérations d'ordre éthique. L'évolution a été la même, la laïcisation s'y ajoutant, s'agissant de nos systèmes d'abattage occidentaux. Les catégories de licite/illicite, de légal/illégal - elles ne sont guère différentes sur le fond -, organisent alors le processus technique. Dans le contexte moderne, l'abattage rituel, le fait de manger de la viande halâl ou casher, apparaissent de plus en plus exclusivement comme des sortes de «marqueurs d'identité » assurant la cohésion du groupe, comme le fait d'arborer certains traits vestimentaires. C'est bien sûr ces bases que se joue l'identité des groupes et le rejet ou l'acceptation de l'autre dans nos sociétés modernes.

Ceci m’amène à une ultime réflexion. S'il est une notion sur laquelle je m'interroge parmi celles que Leroi-Gourhan a introduites dans le champ de l'analyse technologique, c'est beaucoup plus celle de «style » que celle de «tendance ». Tout est bien sûr question de définitions. Mais il me semble que l'imputation à Leroi-Gourhan de ces présupposés positivistes que j'évoquais précédemment résulte beaucoup plus de son utilisation de la notion de "style » que de celle de « tendance », car elle mêle en fait deux définitions. Je dirais rapidement que le style peut être considéré comme l'ensemble des significations et pratiques irréductibles qu'entraîne dans le système technique l'indissociable relation entre les savoir-faire et les savoir-dire - le sacrifice musulman diffère par exemple du sacrifice « africain »- mais il est aussi ce qui reste irréductible, ce que j'appelais précédemment «marqueur d'identité », dans une situation, telle celle de l'abattage rituel musulman dans la société moderne, où la perte des significations de l'acte technique, des savoir-dire, entamée avec l'islam, trouve son achèvement. La notion de style telle que l'emploie Leroi-Gourhan recouvre ainsi deux réalités d'ordres différents, l'une caractérisée par l'« excès » de sens, l'autre par la " perte » de sens. Si l'on admet, après Marx et bien d'autres, que cette perte de sens accompagne plus particulièrement le processus de technicisation de la société moderne, et correspond à la place, autonome et déterminante, qu'elle accorde à la technique et à ses produits, on comprendra peut-être mieux le sens de cette dernière interrogation. Il y va en effet du statut épistémologique de l'analyse technologique elle-même. 
1. Digard souligne que A. Leroi-Gourhan (1945: 13) prônait une « technologie fondée sur les matières premières », « posant en principe que c'est la matière qui conditionne toute technique et non pas les moyens et les forces » $(1945: 18)$. Il poursuit: « Les animaux constituent toutefois, c'est le moins que l'on puisse dire, une "matière" un peu particulière: une matière vivante, animée, à l'organisation (individuelle et collective) complexe, douée de sens, voire d'intelligence, capable d'autonomie » (Digard 1990 : 202).

2. Exclusion relative certes puisqu'un chapitre de Milieu et Techniques (Leroi-Gourhan 1945) est consacré à la Chasse et un autre à l'Élevage; celui-ci toutefois se borne à une énumération des espèces domestiquées d'ailleurs du plus haut intérêt car elle souligne la conception novatrice qu'avait Leroi-Gourhan de la domestication animale.

3. Je me permets de renvoyer à de précédentes interventions sur ce thème: Bonte (1985, 1986, 1993).

4. Digard (1990 : 255) citant Deforge (1985: 127).

5. Lait, sang, poils, prélevés sur l'animal vivant, monture, utilisation des déchets pour l'engrais, le chauffage, etc.

6. Sinon dans son contexte symbolique, bien sûr, nous le verrons à propos du sacrifice, et dans des travaux récents, celui de N. Vialles sur les abattoirs (1987) ou encore au cours du séminaire organisé sur ce thème par A.-M. Brisebarre dans le cadre de l'équipe APSONAT (Muséum National d'Histoire Naturelle) en 1990-1991 et qui devrait déboucher sur la publication d'un ouvrage.

7. L'étude du contexte symbolique du sacrifice, à laquelle nous ne nous consacrerons pas dans le cadre particulier de ce colloque, met en évidence la continuité cosmique qu'établit l'animal entre les divers ordres, tant l'animal vivant que sa viande dans la chaîne alimentaire, et sa fonction de médiateur entre nature et surnature. Ce système est particulièrement développé dans la pensée indo-européenne. Voir Bruce Lincoln (1986).

8. Recherches de terrain sur des sociétés musulmanes, en Mauritanie, reprises et développées dans le cadre d'un programme collectif sur « Le sacrifice musulman », dirigé par A.-M. Brisebarre et A. Gokalp, initié grâce à un financement du MRT, obtenu en 1990, et relayées dans le cadre du GOR 745, «Anthropologie comparative des sociétés musulmanes » (Brisebarre et Gokalp 1993).

9. Le pèlerinage, la prière, le jeûne, l'aumône légale.

10. Sourate 2. La Génisse: «Voici il vous interdit la charogne, le sang, la viande de porc et ce qui est immolé pour tout autre qu'Allah» (vers 173-175).
11. Sur ce point voir Chelhod (1955).

12. On pourrait développer l'opposition entre la technique de l'égorgement et celle de la lapidation où l'on peut voir une opposition structuralement significative.

13. Outre la sourate «La Génisse » précédemment citée, celle sur « La Table », « Les Troupeaux », etc.

14. Un an pour les ovins et caprins, quatre ans pour les bovins et six ans pour les camelines.

15. Interrompant brutalement l'arrivée d'air et de sang au cerveau, il provoquerait un collapsus et la mort rapide avec le minimum de souffrance.

16. Pour les chameaux et autres gros animaux (chevaux) une autre technique est prévue et parfois considérée comme valorisée. L'animal est debout, un patte antérieure liée, et l'écoulement de sang est obtenu, avec un poignard et non un couteau, en poignardant la bête au cour, en haut du poitrail, à l'endroit du collier.

17. Tout animal domestique en fuite peut en outre être traité comme du gibier et être tué, d'une flèche par exemple.

18. Seule exception, semble-t-il (al-Bukhãrl), le corps partagé en deux moitiés symétriques, type de sacrifice pratiqué anciennement dans l'aire proche-orientale où il accompagnait souvent les pactes d'alliance.

19. Il suffit qu'il s'agisse d'un objet suffisamment aiguisé pour remplir cette fonction, à condition indiquent toutefois les Hadith, « que ce ne soit pas avec les ongles ou avec les dents. Les ongles servent de couteau aux Abyssins et quant aux dents ce sont des os ».

20. Étude pour laquelle je suis fortement redevable à A.-M. Brisebarre pour ses observations faites dans le cadre du programme, «Le sacrifice musulman », déjà évoqué.

21. Décret n 80-791 du ler octobre 1980 modifié en ce qui concerne l'abattage rituel par le décret n 81-606 du 18 mai 1981 qui concerne aussi bien l'abattage halâl que l'abattage casher.

22. Avec d'autres références, utilisant d'autres moyens infiniment médiatiques, les émissions animées par une célèbre ancienne vedette de cinéma sur la mise à mort de l'animal, n'ont-elles pas pour but de produire un autre champ de codification du licite et de l'illicite en ce domaine de la mort de l'animal: licites les bovins, illicite le cheval, licites les escargots ou les langoustes, illicites les grenouilles, licite le magret de canard, illicite le foie gras d'oie, etc.

23. 1500000 ovins sont sacrifiés chaque année à l'occasion du Pèlerinage mobilisant 300 vétérinaires et 6000 égorgeurs (turcs pour la plupart) dans les abattoirs modernes de Mina équipés pour traiter 5000 sacrifices par heure et pour garder en frigorifique 700000 
carcasses. Les tanneurs de Mazamet, depuis 1987, sous-traitent l'utilisation des peaux. On lira avec intérêt à ce propos l'ouvrage de Slimane Zaghidoui (1989).

24. Communication personnelle A. Gokalp.

25. Chelhod note par ailleurs que, conformément à un principe d'inversion des valeurs assez classique, le sang peut être utilisé pour répondre à des situations de crise: le sang d'un homme noble était ainsi consommé en petite quantité comme une cure contre la rage.

26. C'est paradoxalement cette représentation de la place du sacrifice qui explique l'importance qu'il prend dans les formes locales et populaires de l'islam, qui intègrent des pratiques sacrificielles souvent antérieures à l'islamisation (culte des saints, zar, etc.).
27. De cette racine dérive le toponyme Mina, lieu sacré où ont lieu les sacrifices lors du Pèlerinage à La Mecque, le terme maniyya, la mort, mais aussi mani, le sperme et muniya, le désir.

28. Si, depuis, l'analyse ethnologique des techniques s'est affirmée " technologie culturelle», abondant en ce sens, elle gagnerait encore à s'articuler plus étroitement avec un autre domaine de recherches dont l'origine, dans le mouvement des idées anthropologiques, est autre que celle de la technologie: je veux parler des « ethnosciences».

\section{RÉFÉRENCES}

Bonte, P. 1984 Il Bestiamo produce gli Uomini. Valore et feticismo des bestiamo nell' Africa orientale, Studi Storici 4: 875-896.

- 1985 Faits techniques et valeurs sociales: quelques directions de recherche, Techniques et culture 5 : 19-48.

— 1986 Les Recherches ethnologiques sur les techniques en France après A. Leroi-Gourhan, La Pensée 254: 34-47.

— 1993 Après Leroi-Gourhan? Tendances et problèmes d'une approche ethnologique des faits techniques, Nouvelles de l'Archéologie 48-49: 18-22.

Brisebarre A.-M. \& A. Gokalp 1993 Le Sacrifice musulman. Espaces et temps d'un rituel. Paris: Ministère de l'Enseignement supérieur et de la Recherche (Action concertée « Anthropologie »).

Chelhod, J. 1955 Le Sacrifice chez les Arabes. Paris: Presses Universitaires de France.

Conte, E. 1994 Choisir ses parents dans la société arabe. La situation à l'avènement de l'islam In P. Bonte (dir.) Épouser au plus proche. Inceste, prohibitions et stratégies matrimoniales autour de la Méditerranée. Paris: Éditions de l'EHESS: 165-187.

Deforge, Y. 1985 Technologie et génétique de l’objet industriel. Paris: Maloine (« Université de Compiègne »). Digard, J.-P. 1990 L'Homme et les animaux domestiques. Anthropologie d'une passion. Paris: Fayard.

Detienne M. \& J.-P. Vernant 1979 La Cuisine du sacrifice en pays grec. Paris: Gallimard.

Evans-Pritchard, E.E. 1956 Nuer religion. Oxford: Clarendon Press.

Leroi-Gourhan, A. 1945 L'Homme et la matière. Paris: Albin Michel.

— 1945 Milieu et Techniques. Paris: Albin Michel.

Lincoln, B. 1986 Myth, cosmos and society. Indo-European themes of creation and destruction. Cambridge (Mass.) : Harvard University Press.

Robertson Smith, W. 1972 [1889] The Religion of the semites. The Fundamentallnstitutions. New York: Scocken Books.

Vialles, N. 1987 Le Sang et la chair. Paris: Éditions de la Maison des Sciences de l'Homme/Ministère de la Culture et de la Communication.

Zaghidoui, S. 1989 La Vie quotidienne à La Mecque de Mahomet à nos jours. Paris: Hachette. 


\section{RÉSUMÉ}

Quand le rite devient technique. Sacrifice et abattage rituel dans le mode musulman. La notion de tendance quand elle s'applique au traitement technique de la matière vivante, en l'occurrence la domestication de l'animal et la consommation de sa viande, conserve-t-elle sa valeur heuristique? L'analyse s'emploie à le démontrer, mais tout en soulignant que ceci nécessite qu'elle se dégage de tout postulat positiviste et qu'elle associe l'étude des techniques à celle des classifications. En islam l'abattage rituel, associé à la notion de sacrifice de l'animal, exclusif de toute autre forme de consommation des viandes met en jeu les catégories de licite et d'illicite, et implique au-delà un système complexe de représentations du règne animal (et de l'homme). La technicisation de l'acte rituel dans les abattoirs permet la comparaison avec le modèle occidental de mise à mort de l'animal.

\section{ABSTRACT}

When ritual becomes tecnology. Sacrifice and rital slaughter in the Muslim world. Does the notion of tendency still preserve its heuristic value when it is applied to living matter, in this case the domestication of the animal and the consuming of its? The analysis attempts to demonstrate the truth of this proposition, but in emphasizing that is necessary to slough off positivistic postulates and to associate the study of technics with that of classifications. In Islam ritual slaughter associated wiht the idea of the sacifice of the animal regardless the way in which the meat is cosumed brings into play the categories of licit and illicit, and beyond that a complex system of representations of the animal kingdom (and of man). The industrialization of technics in the slaughter-houses of the ritual act leads to a comparison of the act of killing with the Western model.

\section{MOTS CLÉS}

Rite, sacrifice, animal, islam.

\section{KEYWORDS}

Ritual, sacrifice, animal, islam.

\section{NOTES ET RÉFÉRENCES DE L'INTRODUCTION}

Photo d'ouverture: Dakar, Tabaski, 2001. Toute la famille participe au sacrifice du mouton avec des rôles distincts. 